\title{
Insuficiência Adrenal Relativa e Uso de Corticosteróides na Sepse: Estamos mais Próximos de um Consenso?
}

\author{
Relative Adrenal Failure and the Use of \\ Steroids in Sepsis: Are we Closer to a Consensus?
}

$\mathrm{O}$ corticosteróides são utilizados há mais de 60 anos como tratamento adjunto das infecções, tendo como principal objetivo atenuar a resposta inflamatória local e sistêmica'. Seu uso em pacientes críticos já foi avaliado em diversos cenários e em doses distintas, havendo uma restrição quanto a sua utilização após a evidência de aumento da mortalidade em um estudo aleatório publicado no final dos anos 80 2. Mais recentemente, na década de 1990, observamos um número expressivo de estudos demonstrando benefícios do uso de corticosteróides em pacientes com choque séptico ${ }^{3}$ onde se observou inicialmente reversão do choque ${ }^{4,5}$, atenuação dos indicadores de resposta inflamatória sistêmica (citocinas e PCR) ${ }^{6-8}$ e por fim redução expressiva da mortalidade ${ }^{9}$. Tais estudos surgiram atrelados ao crescente conhecimento das complexas alterações neuroendócrinas presentes em pacientes criticamente enfermos ${ }^{10-13}$ e a demonstração da presença de insuficiência adrenal relativa em um percentual elevado de pacientes com sepse ${ }^{14-16}$. Embora a presença de disfunção adrenal em pacientes críticos com sepse seja conhecida desde o início do século $X X^{17}$, ainda hoje há controvérsia acerca do diagnóstico de insuficiência adrenal em pacientes criticamente enfermos ${ }^{18}$. Nestes, a dosagem do cortisol sérico $<18 \mu \mathrm{g} / \mathrm{dL}$ pode confirmar o diagnóstico de insuficiência adrenal ${ }^{18}$. Entretanto, o teste diagnóstico mais empregado é o teste de estimulação rápida com ACTH (p. ex.: Cortrosina ${ }^{\circledR}$, Synachten $^{\circledR}$ ) que tem como principal limitação a dose utilizada para estimular a secreção de cortisol, uma vez que é realizada com doses suprafisiológicas de ACTH $(250 \mu \mathrm{g})$ e o teste com doses menores $(1 \mu \mathrm{g})$ ainda não está padronizado. Outros testes dinâmicos utilizados em pacientes com suspeita

Jorge Ibrain Figueira Salluh,MD, MSC

Unidade de Terapia Intensiva- Hospital Barra D'or, Rio de Janeiro, Brasil

Unidade de Terapia Intensiva- Instituto Nacional de Câncer, Rio de Janeiro, Brasil

E-mail- jorgesalluh@yahoo.com.br

Anna Gabriela Fuks, MD, MSC

Endocrinologia- Hospital Copa D'or, Rio de Janeiro, Brasil de insuficiência adrenal crônica, como o teste de tolerância à insulina e teste da metirapona não devem ser utilizados em pacientes instáveis hemodinamicamente. Outra questão pertinente é que o cortisol dosado é aquele que circula ligado as proteínas e os pacientes críticos freqüentemente encontram-se hipo-albuminêmicos. Nestes casos, o ideal seria dosar o cortisol livre no soro, método extremamente complexo e laborioso e por este motivo não disponível rotineiramente ${ }^{19}$. Contudo se relevarmos os, pertinentes, questionamentos acerca da validade do diagnóstico de insuficiência adrenal em pacientes com sepse algumas evidências contundentes nos parecem hoje irrefutáveis. Em primeiro lugar, ensaios clínicos bem desenhados demonstraram inequivocamente a redução da resposta inflamatória ${ }^{7,8}$, a reversão do choque ${ }^{5,8}$ e a melhora da sobrevida de pacientes com sepse tratados com corticosteróides em baixas doses ${ }^{5,9}$. Tais benefícios devem ser enfatizados pela simplicidade e baixíssimo custo do tratamento. Acrescenta-se a isso o fator segurança demonstrado ao observarmos que mesmo pacientes sem o diagnóstico confirmado de insuficiência adrenal quando tratados com corticosteróides em baixas doses não parecem ter aumento significativo de mortalidade $^{15}$. É, contudo, necessária alguma cautela em meio a tamanho entusiasmo. Primeiramente, a dose total diária e o tempo de administração de corticosteróides na sepse ainda estão por ser determinado. Sabe-se, contudo, que o uso de doses entre 200 a 300 mg de hidrocortisona por dia,9, associados ou não a fludrocortisona, por 7 a 10 dias $^{9}$ com um esquema de redução rápida das doses dentro desse período de tempo ${ }^{7,8}$ parece ser suficiente para o tratamento de pacientes sépticos. Algumas questões ainda ficam em aberto e dizem respeito à forma de administração (infusão contínua ou intermitente) e a necessidade do retorno da infusão em pacientes em que após 7-10 dias de uso voltam a ficar sépticos ou hipotensos. Tais questionamentos devem ser respondidos, ainda que parcialmente, por novos estudos incluindo um ensaio clínico multicêntrico fase III em andamento ${ }^{20}$. 
A aderência a estes protocolos de infusão tem por objetivo primordial evitar o uso excessivo de corticosteróides e seus efeitos colaterais mais proeminentes em pacientes críticos tais como imunossupressão, miopatia e hiperglicemia ${ }^{21}$. Tendo em vista tais complicações, deve haver atenção especial a implementação de protocolos para o controle intensivo da glicemia em pacientes críticos com sepse e em especial naqueles em uso de corticosteróides.

Em resumo é novamente a hora de usar corticosteróides em baixas doses para pacientes com sepse grave e estes devem fazer parte do parco arsenal terapêutico em uma condição clínica de tão elevada mortalidade. Claro está que seu uso deve ser precoce, em baixas doses e por tempo curto. Só assim seus potenciais benefícios poderão superar os intimidantes efeitos adversos que reprovaram seu uso no passado.

\section{REFERÊNCIAS}

01. Perla D, Marmorston $\mathrm{J}$ - Suprarenal cortical hormone and salt in the treatment of pneumonia and other severe infections. Endocrinology, 1940:27:367-374.

02. Bone RC, Fisher CJ Jr, Clemmer TP et al - A controlled clinical trial of high-dose methylprednisolone in the treatment of severe sepsis and septic shock. N Engl J Med, 1987;317:653-658.

03. Annane D, Bellissant E, Bollaert PE et al - Corticosteroids for severe sepsis and septic shock: a systematic review and meta-analysis. BMJ, 2004;329:(7464):480.

04. Bollaert PE, Charpentier C, Levy B et al - Reversal of late septic shock with supraphysiologic doses of hydrocortisone. Crit Care Med, 1998:26:645-650.
05. Briegel J, Forst H, Haller M et al - Stress doses of hydrocortisone reverse hyperdynamic septic shock: a prospective, randomized, double-blind, single-center study. Crit Care Med, 1999;27:723-732.

06. Confalonieri M, Urbino R, Potena A et al - Hydrocortisone infusion for severe community-acquired pneumonia: a preliminary randomized study. Am J Respir Crit Care Med, 2005;171:242-248.

07. Keh D, Boehnke T, Weber-Cartens $\mathrm{S}$ et al - Immunologic and hemodynamic effects of "low-dose" hydrocortisone in septic shock: a doubleblind, randomized, placebo-controlled, crossover study. Am J Respir Crit Care Med, 2003;167:512-520.

08. Oppert M, Schindler R, Husung C et al - Low-dose hydrocortisone improves shock reversal and reduces cytokine levels in early hyperdynamic septic shock. Crit Care Med, 2005;33:2457-2464.

09. Annane D, Sebille V, Charpentier $C$ et al - Effect of treatment with low doses of hydrocortisone and fludrocortisone on mortality in patients with septic shock. JAMA, 2002;288:862-871.

10. Rothwell PM, Udwadia ZF, Lawler PG - Cortisol response to corticotropin and survival in septic shock. Lancet, 1991;337:582-583.

11. Beishuizen A, Thijs LG - The immunoneuroendocrine axis in critical illness: beneficial adaptation or neuroendocrine exhaustion? Curr Opin Crit Care, 2004;10:461-467.

12. Aberdein J, Singer M - Clinical review: a systematic review of corticosteroid use in infections. Crit Care, 2005;10:203.

13. Vanhorebeek I, Van den Berghe G - The neuroendocrine response to critical illness is a dynamic process. Crit Care Clin, 2006;22:1-15.

14. Salluh JI, Verdeal JC, Mello GW et al - Cortisol levels in patients with severe community-acquired pneumonia. Intensive Care Med, 2006;28:1-4

15. Annane D, Sebille V, Troche $G$ et al - A 3-level prognostic classification in septic shock based on cortisol levels and cortisol response to corticotropin. JAMA, 2000;283:1038-1045.

16. Salgado D, Paiva R, Rodrigues $\mathrm{M}$ et al - Adrenal failure in neurological and septic critically ill patients. Crit Care, 2005;9:(Suppl1):P399.

17. Waterhouse R - A case of suprarenal apoplexy. Lancet, 1911;1:577578.

18. Annane D, Bellissant E, Cavaillon JM - Septic shock. Lancet, 2005;365:(9453):63-78.

19. Hamrahian AH, Oseni TS, Arafah BM - Measurements of serum free cortisol in critically ill patients. N Engl J Med, 2004;350:1629-1638.

20. Corticus Study -http://www.clinicaltrials.gov/show/NCT00147004

21. Latronico N, Peli E, Botteri M - Critical illness myopathy and neuropathy. Curr Opin Crit Care, 2005;11:126-132. 